\title{
Lamb Waves and Nearly-Longitudinal Waves in Thick Plates
}

\author{
David W. Greve ${ }^{\mathrm{a}}$, and Irving J. Oppenheim ${ }^{\mathrm{b}}$, and Peng Zheng ${ }^{\mathrm{c}}$, \\ ${ }^{a}$ Dept. of Electrical and Computer Engineering, Carnegie Mellon University, Pittsburgh, PA 15213 \\ ${ }^{\mathrm{b}}$ Dept. of Civil and Environmental Engineering, Carnegie Mellon University, Pittsburgh, PA 15213 \\ ${ }^{\mathrm{c}}$ Dept. of Physics, Carnegie Mellon University, Pittsburgh, PA 15213
}

\begin{abstract}
We discuss waves created in relatively thick plates by edge excitation at frequency-thickness (fd) products that correspond, in principle, to multiple Lamb wave modes. For relatively low values of the fd product it is clear that Lamb wave modes will be generated, while at large values of the fd product we observe a bulk (longitudinal wave) in the solid, but influenced by reflections from the plate surfaces. We show that for a range of intermediate fd products a train of regularly spaced nearly-longitudinal waves is generated. The development of a lead pulse and trailing pulses, all traveling at the longitudinal (bulk) wave speed, is well known and has been explained in the literature. In this paper we describe the transition from Lamb wave generation to the formation of nearly-longitudinal waves with their trailing pulses. We report experimental results and theoretical results, with good correspondence between them. We also examine the transfer of energy from leading to trailing pulses, which means that such nearly-longitudinal waves will not propagate indefinitely; however, we show that they retain ample energy for flaw detection at distances of several meters. Most importantly, we study the interaction of these trailing pulses with cracks, again showing experimental results and theoretical predictions that are consistent with one another. The results suggest that these nearly-longitudinal waves are an attractive option for flaw detection because of their shorter wavelength (as compared to Lamb waves at low fd products) and because they preserve their pulse train characteristics after scattering.
\end{abstract}

Keywords: Flaw detection, Lamb waves, longitudinal waves, plates, pulse-echo, ultrasonics.

\section{INTRODUCTION}

Extensive research has been directed at the detection of defects in plate-like structures using Lamb waves. This application has been motivated by the fact that pulses formed from Lamb waves propagate with low attenuation for considerable distances in plates. However, the existence of multiple Lamb wave modes presents a significant complication. Lamb waves are typically excited by transducers mounted on the surface of the plate; numerous modes are present when the frequency-thickness product fd exceeds, say, $3 \mathrm{MHz}-\mathrm{mm}$ (in metals such as steel or aluminum). As a result, many researchers have recommended working at low fd products so that only a small number of modes are excited [1] and other work has concerned the development of transducers that selectively emit particular modes $[2,3]$. However, limiting operation to low frequencies is not a completely satisfactory solution because scattering becomes weaker and the resolution is degraded when the defect is small compared to the wavelength.

We have studied the creation of waves in plates by edge excitation [4] at fd products that correspond to multiple Lamb wave modes; in this paper we briefly summarize those results and present studies of flaw detection. For relatively low fd it is clear that Lamb wave modes will be generated while at large fd we will encounter longitudinal waves, possibly influenced by reflections from the surface. We show that for a range of intermediate fd there develops a pulse train of nearly longitudinal waves. These "trailing pulses" are well known and have been discussed in the literature. We then consider the interaction of these trailing pulses with flaws such as cracks. Results suggest that these nearly longitudinal waves are an attractive option for flaw detection.

* Contact author: ijo@cmu.edu; 412-268-2950; Carnegie Mellon University, Department of Civil and Environmental Engineering, Pittsburgh, PA, 15213. 


\section{EXCITATION OF PLATE EDGE}

We consider waves generated by a force applied normal to the edge of a plate. Figure 1 shows the geometry under consideration. A plate of thickness $d$ is excited by a distributed force normal to the edge. A good approximation to this excitation is obtained when the plate is excited by a conventional piezoelectric transducer using grease coupling.

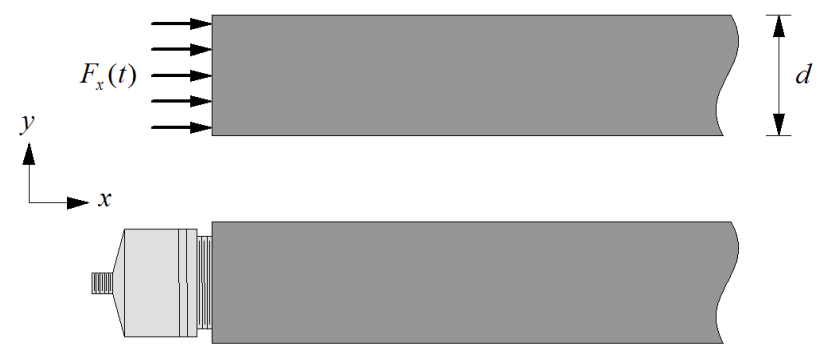

Figure 1. Ideal excitation by force normal to edge (top) and practical case of edge excitation (bottom) by grease-coupled transducer

We first consider the transition from a low-frequency regime, in which only Lamb waves are understood to propagate, to a high-frequency regime in which only longitudinal waves would be generated. For plates with thicknesses typical of structural and aerospace applications, we will show that the frequency need not be very high (the fd product need not exceed $15 \mathrm{MHz}-\mathrm{mm}$ ) in order to create nearly-longitudinal waves that propagate for considerable distances. We also obtain insight into the formation of trailing pulses that are known to form when longitudinal waves propagate in plates.

The trailing pulses that form when a nearly-longitudinal wave is excited in a plate are shown experimentally in Figure 2. We excited a steel plate, $19 \mathrm{~mm}$ thick and $30.5 \mathrm{~cm}$ long, by an edge-mounted piezoelectric wafer (Piezo Systems 5A4E), $15 \times 15 \times 1 \mathrm{~mm}$ with a lead backing $3 \mathrm{~mm}$ thick, bonded to the plate edge with cyanoacrylate adhesive. The transducer was driven by a five-cycle windowed sinusoid voltage waveform

$$
v(t)=\left\{\begin{array}{cr}
v_{0} \sin (2 \pi f t) \cdot\left(\sin \left(\frac{f t}{20 \pi}\right)\right)^{2} & t<\frac{5}{f} \\
0 & t \geq \frac{5}{f}
\end{array}\right.
$$

with a center frequency of $2.2 \mathrm{MHz}$. Figure 2 shows the signal received at a similar transducer on the opposite plate edge, consisting of a pulse at $52.6 \mu$ s followed by several trailing pulses. The first arrival at $52.6 \mu$ s corresponds to a group velocity of $5.8 \mathrm{~km} / \mathrm{s}$, which agrees well with the longitudinal velocity in steel. The leading pulse is followed by several trailing pulses, equally spaced at roughly $5 \mu \mathrm{s}$. In this instance the leading pulse is the largest, but at different propagation distances or at different center frequencies one of the trailing pulses may be the largest.

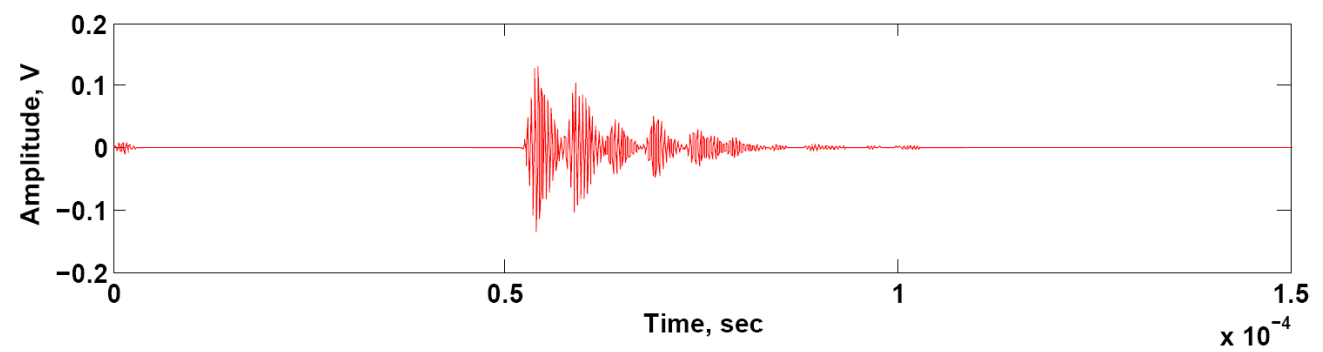

Figure 2. Signal received after a propagation distance of $30.5 \mathrm{~cm}$; the small signal at $0 \mathrm{~s}$ is due to electrical stray coupling 
Trailing pulses are well known [5] and are associated with boundary reflections; the pulses have been termed "secondary echoes produced by split-off transverse waves" and explained as shown in Figure 3. In general, the transducer creates a longitudinal wave that propagates straight down the plate (a) and a longitudinal wave that can be viewed as striking the plate edge at oblique incidence (b). The latter wave produces a reflected longitudinal wave (c) and a reflected transverse wave (d). Consider now the case of the oblique longitudinal wave (b) approaching the boundary at grazing incidence, such that the reflected longitudinal wave (c) propagates straight down the plate; in that case the reflected transverse wave (d) forms at the critical angle $\theta$, and when it strikes the opposite edge it creates another longitudinal wave (e) propagating straight down the plate but delayed with respect to the original pulse (a), along with a reflected transverse wave (f). The process repeats, resulting in additional trailing longitudinal pulses propagating straight down the plate.

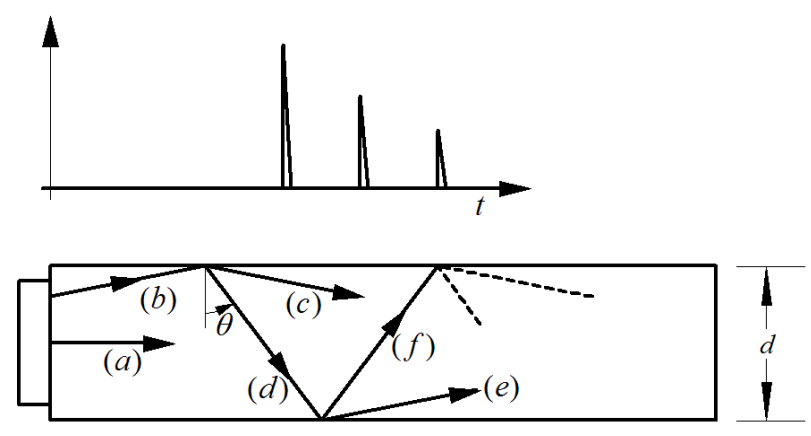

Figure 3. Simple model [5] for formation of trailing pulses as "split-off transverse waves"

In steel, the reflected transverse wave (d) is produced with an angle $\theta$ near $33^{\circ}$, and the time separation between successive trailing pulses, $\Delta \mathrm{t}$, can be calculated as

$$
\Delta t=\frac{d}{c_{t} \cos \theta}-\frac{d \tan \theta}{c_{l}}
$$

where $c_{t}$ and $c_{1}$ are the transverse and longitudinal wave velocities, respectively. In steel, at a plate thickness of $19 \mathrm{~mm}$, $\Delta \mathrm{t}$ is predicted to be $4.7 \mu \mathrm{s}$, which is in reasonable agreement with the $\Delta \mathrm{t}$ of $5 \mu \mathrm{s}$ observed experimentally in Figure 2 .

However, this simple, classical picture provides no guidance as to the lowest fd at which the longitudinal pulse propagates for useful distances, nor does it determine how rapidly the leading pulse decays in amplitude. In the following section, we report on our investigations of the generation and propagation of waves in this geometry using finite element simulations. These results provide insight into the generation and propagation of trailing pulses. In addition, these simulations show how a propagating pulse sheds energy by the creation of trailing pulses with an identical shape.

\section{SIMULATIONS OF WAVE GENERATION}

Simulations were performed in the Comsol 3.2 plane-strain structural mechanics mode for a plate $2 \mathrm{~cm}$ thick and $35 \mathrm{~cm}$ long, and a force in the $\mathrm{x}$ direction with the time dependence of Eq. (1) was applied to the left edge of the model. Material parameters of $200 \mathrm{GPa}$ elastic modulus, 0.33 Poisson ratio, and 7.85 specific gravity were used, appropriate for steel. A transient (time dependent) analysis was performed using a step size of 1/10f.

We first consider the effect of pulse center frequency on wave generation. Figure 4 shows the effect of launching a wave with edge excitation at various frequencies by plotting the particle displacement (in the $\mathrm{x}$ direction) in the plate at $50 \mu \mathrm{s}$. At low frequencies (up to about $450 \mathrm{kHz}$ ) the wavefront velocity is significantly slower than the bulk wave longitudinal velocity, and particle displacements are similar to those for low-order symmetric Lamb modes. Figure 5 shows a snapshot of particle velocity at $175 \mu$ s in a plate $85 \mathrm{~cm}$ long. Two symmetrical Lamb wave modes are visible, a faster mode with highest particle velocity at the top and bottom surfaces, and a slower mode with highest particle velocity at the midthickness. The group velocity for the faster mode, based upon the wavefront position, is estimated as $4.6 \mathrm{~km} / \mathrm{s}$, 
and the group velocity for the slower mode is estimated as $3.4 \mathrm{~km} / \mathrm{s}$. These particular modes can be identified as $\mathrm{S} 1$ and $\mathrm{S} 2$ modes, respectively, and both of these Lamb wave modes are predicted to be present at an fd product of $4 \mathrm{MHz}-\mathrm{mm}$.

At higher fd products, theory predicts that a greater number of Lamb wave modes will be present with a considerable range of group velocities, and as a result one might expect a long pulse train with complex character. However, the simulation results in Figure 6 for higher frequency excitations show particle velocities that are nearly uniform across the thickness of the plate, traveling with a group velocity essentially equal to the bulk wave longitudinal velocity. This behavior is fully established at $750 \mathrm{kHz}$ and above (when the fd product reaches $15 \mathrm{MHz}-\mathrm{mm}$ ). The trailing portion of the response differs in character from a trailing region of Lamb wave modes.

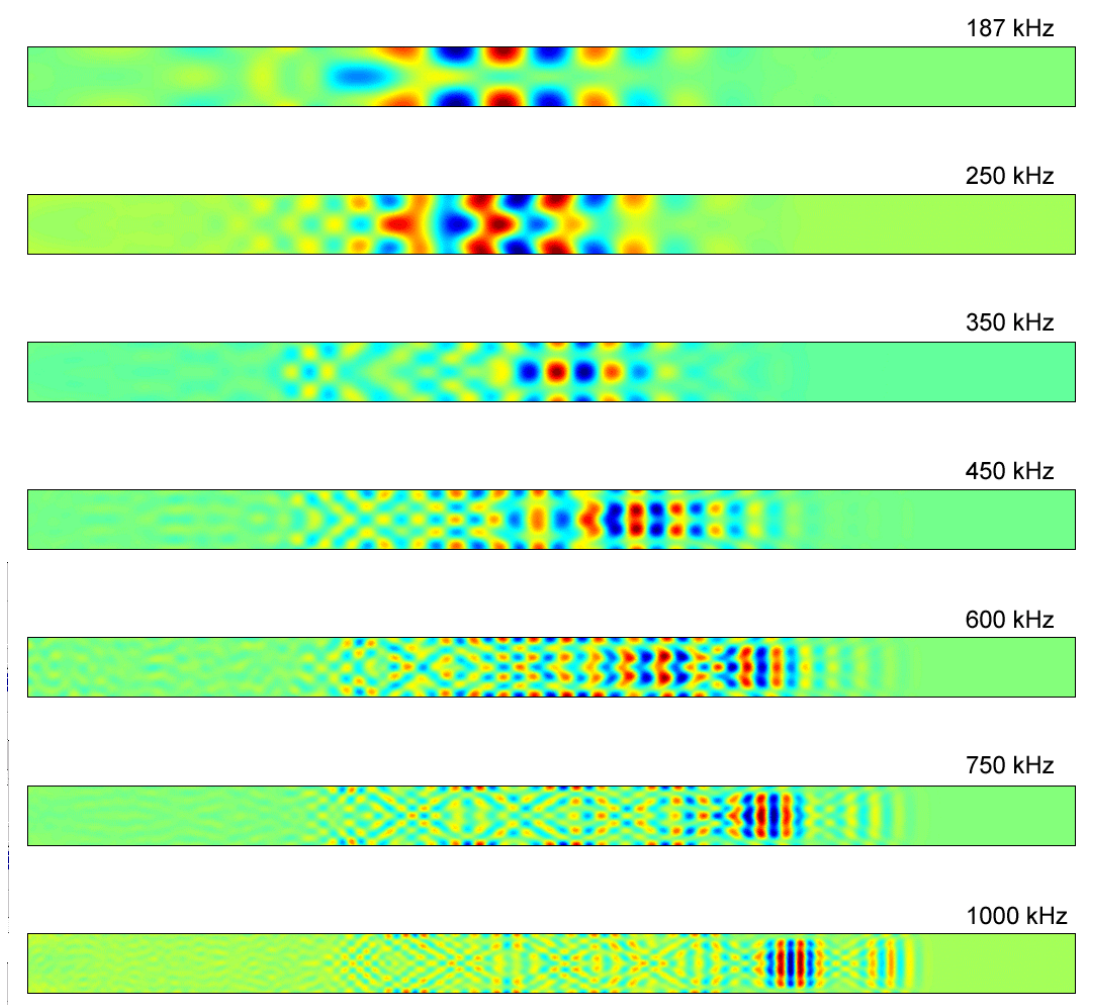

Figure 4. Effect of center frequency on propagating waves for a steel plate $2 \mathrm{~cm}$ thick; color shows particle x-displacement at $50 \mu \mathrm{s}$.

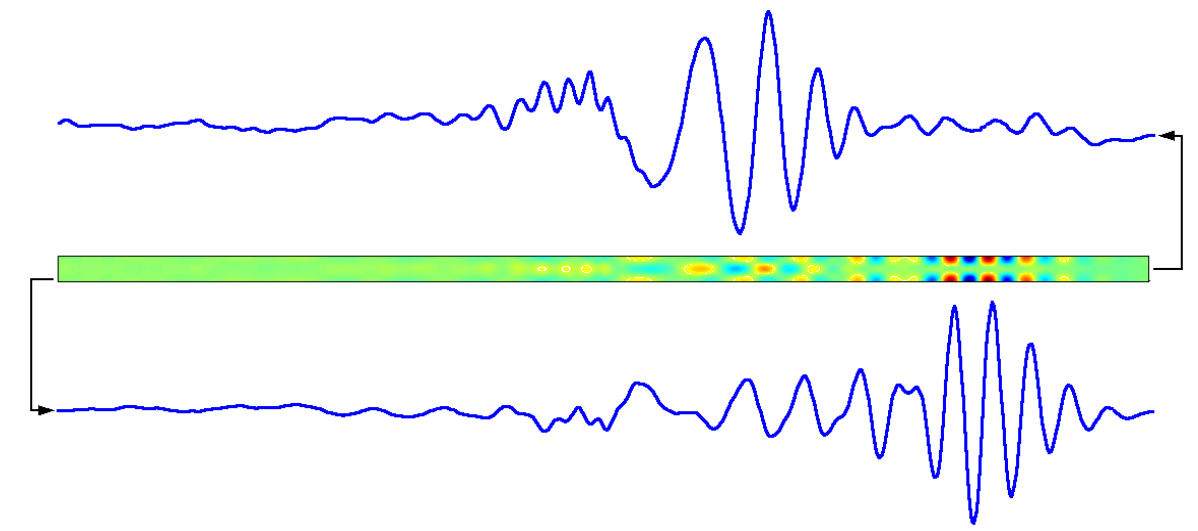

Figure 5. Snapshot at $175 \mu$ s, center frequency $200 \mathrm{kHz}$, showing x displacement at midthickness (top) and at surface (bottom) 


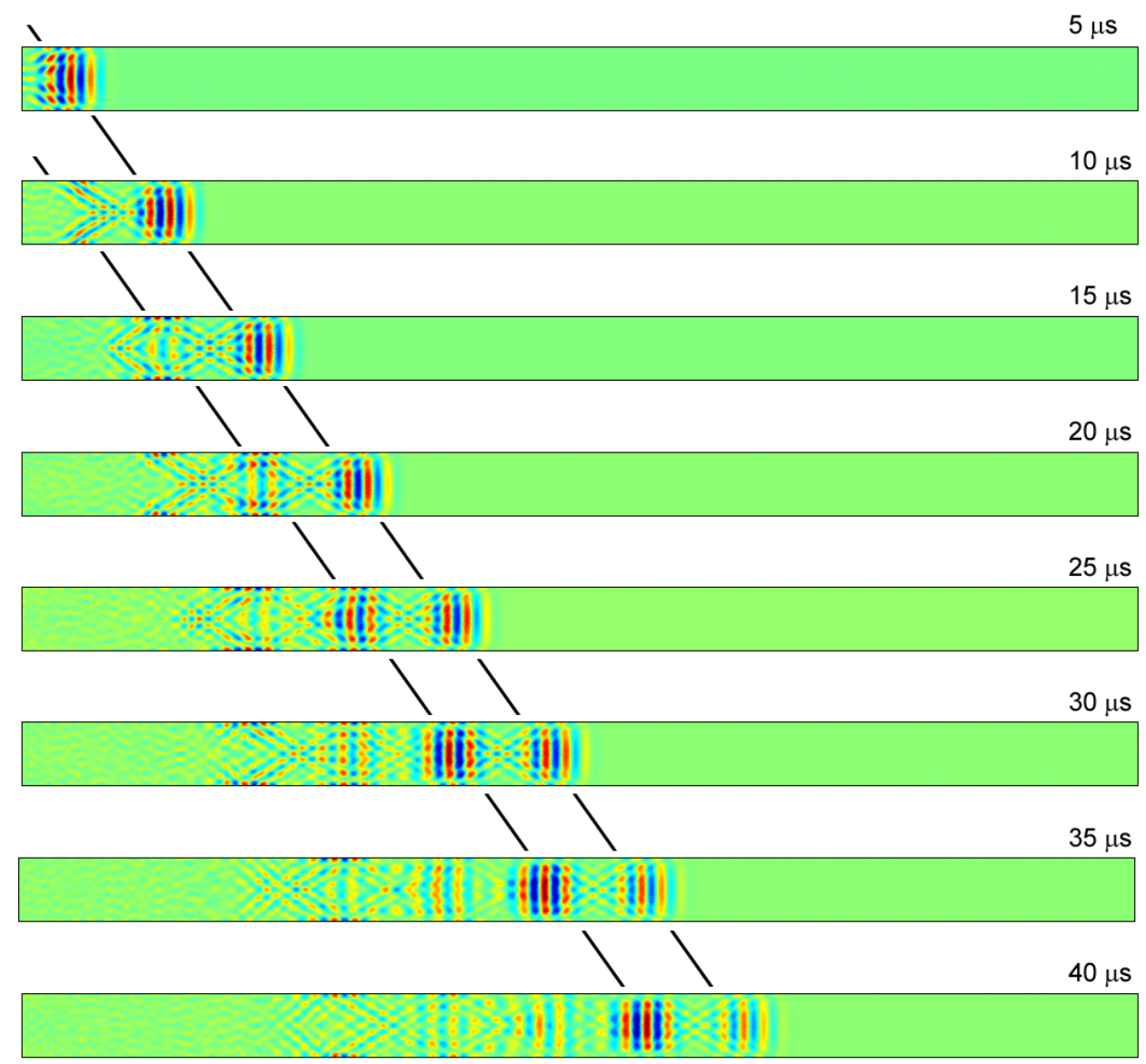

Figure 6. Snapshots showing $\mathrm{x}$ displacement in steel plate, $2 \mathrm{~cm}$ thick, $1 \mathrm{MHz}$ center frequency

Figure 6 shows snapshots of particle displacement in the $\mathrm{x}$ direction at different times for a $1 \mathrm{MHz}$ excitation of a plate $20 \mathrm{~mm}$ thick. The simulations show the formation of a near-planar wavefront within the first 5-10 $\mu$ s of propagation. This wavefront propagates at the longitudinal velocity and gradually loses energy while a second trailing pulse forms and gains energy. Elsewhere we show [4] that the transfer of energy from any pulse to its trailing pulses is dependent on plate thickness, center frequency, and time (path length).

\section{SCATTERING FROM FLAWS, SIMULATION STUDY}

We next consider scattering of nearly-longitudinal waves from a crack. It is possible that scattering from a crack would result in mode conversion to multiple Lamb wave modes, which would make detection of cracks substantially more difficult. Therefore, we undertook simulation and experimental studies to determine whether such unwanted mode conversion occurs. In order to observe such mode conversion, were it to occur, simulations were performed to include the effect of scattering from a part-thickness slot $1 \mathrm{~mm}$ wide and with varying depth. The simulations were performed for a five-cycle windowed sinusoid excitation (Eq. 1) with a center frequency of $1 \mathrm{MHz}$. The plate was $0.4 \mathrm{~m}$ long, and the crack was located $0.2 \mathrm{~m}$ from the left edge where the excitation was applied. Figure 7 (top) shows the pulse train approaching the crack at $30 \mu \mathrm{s}$, and (bottom) the transmitted and reflected waves after passing the crack at $50 \mu \mathrm{s}$. 

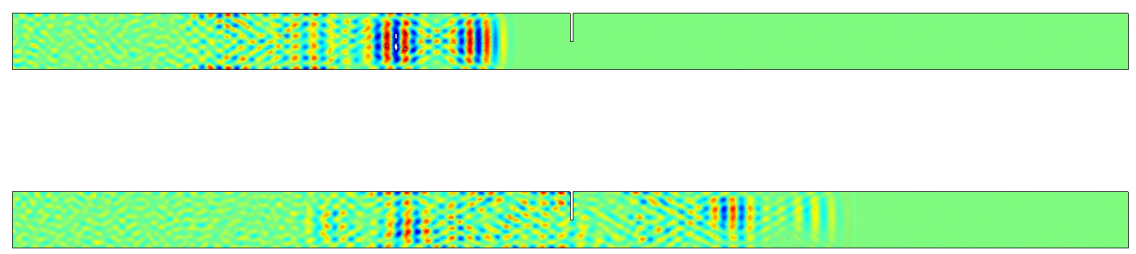

Figure 7. Pulse train, center frequency $1 \mathrm{MHz}$, with a half-thickness crack $1 \mathrm{~mm}$ wide: (top) $t=30 \mu \mathrm{s}$; (bottom) $t=50 \mu \mathrm{s}$ ).

Figure 8, top and bottom, shows the time history of x-direction particle velocity (averaged across the plate thickness) at two locations, $0.10 \mathrm{~m}$ and $0.30 \mathrm{~m}$ from the left edge, which are symmetrically positioned with respect to the crack. Figure 8 (top) shows the incident wave approaching the first location at approximately $17 \mu$ s, and a wave reflected from the crack approaching that same location on its return path at approximately $51 \mu$ s. Figure 8 (bottom) shows the transmitted wave, after passing the slot, approaching the second location at approximately $51 \mu$ s with nearly the same amplitude and shape as the reflected wave returning to the first location. Examining the wave arriving at the second location, and its counterpart reflected wave returning to the first location, we note that the second pulse is larger than the leading pulse, consistent with the simulations for a total travel distance of $0.30 \mathrm{~m}$ without a crack as shown in Figure 8 .

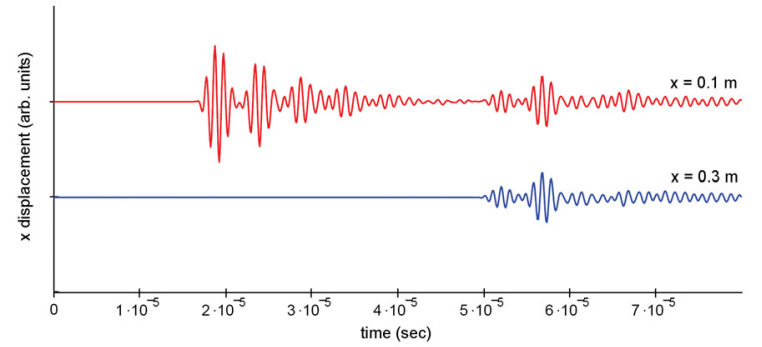

Figure 8. Time history of simulated x-direction particle velocity at two locations

\section{SCATTERING FROM FLAWS, EXPERIMENTAL STUDIES}

Experiments confirm that nearly-longitudinal waves retain their most-important characteristics (velocity, trailing pulse train, and pulse shape) when reflected from a flaw. Figure 9 shows the response recorded at the exciting transducer for a steel plate $19 \mathrm{~mm}$ thick and $30.5 \mathrm{~cm}$ long; a slot $12.6 \mathrm{~cm}$ wide and $5.7 \mathrm{~mm}$ deep has been machined into the plate midway along the length. A nearly-longitudinal pulse train returns to the transducer as an echo from the crack, arriving at roughly $50 \mu \mathrm{s}$. Another pulse train approaches the transducer starting at roughly $100 \mu \mathrm{s}$; it consists of the original wave reflecting from the opposite edge and twice scattered, together with the wave first reflected from the crack being reflected from the transducer edge and then a second time from the crack. The two wave trains are quite similar and show the same characteristics of nearly-longitudinal waves. From the simulations and experiments we conclude that nearly-longitudinal waves retain their velocity and pulse shape when scattered.

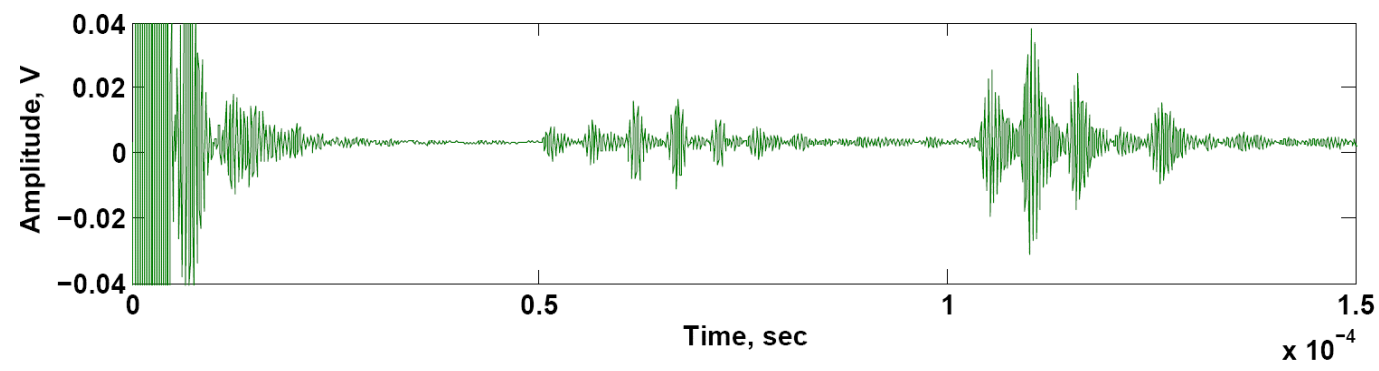

Figure 9. Signal at pulse-echo transducer showing an echo near $50 \mu$ s from the slot, and an echo near $100 \mu$ s from the far edge 
Related experiments were conducted on a steel plate specimen $91.5 \mathrm{~cm}$ long and $9.5 \mathrm{~mm}$ thick, with a through-thickness crack (slot) $3.2 \mathrm{~cm}$ long located $30.5 \mathrm{~cm}$ from the right plate edge. A Krautkramer gamma series $5.0 \mathrm{MHz}$ probe was used, for a nominal fd product of $47.5 \mathrm{MHz}-\mathrm{mm}$, with a USPC-2100 pulser and data acquisition system. Figure 10 is a sketch of the test configuration, and Figure 11 (a through d) shows pulse trains returned as echoes to that transducer.

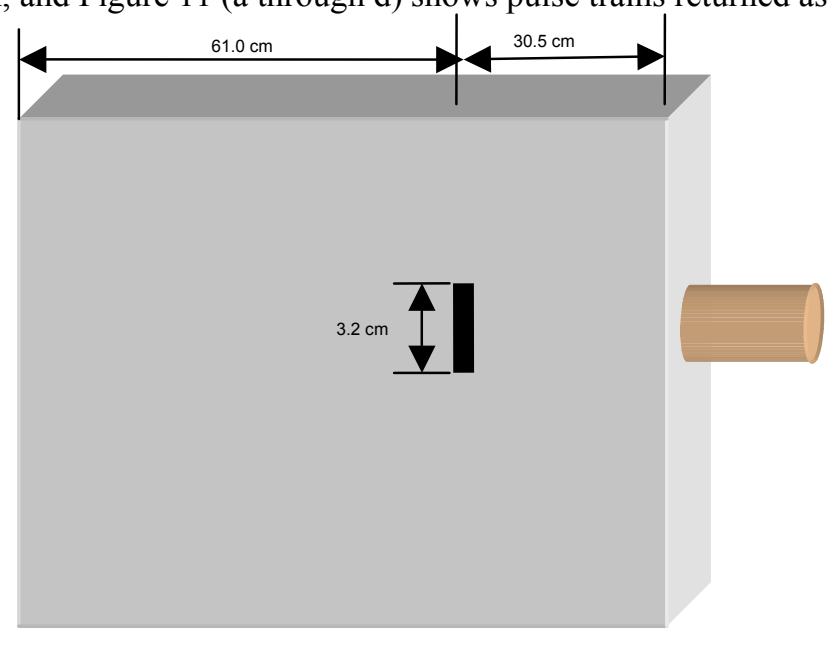

Figure 10. Pulse-echo test, $9.5 \mathrm{~mm}$ plate thickness, $5 \mathrm{MHz}$ commercial transducer (12.5 $\mathrm{mm}$ aperture), through-thickness crack

Figure 11a shows the first echo arriving near $103 \mu \mathrm{s}$, which is consistent with the total path length of $61 \mathrm{~cm}$ and a bulk wave velocity of $5.9 \mathrm{~km} / \mathrm{s}$. The leading pulse is already diminished in comparison the first trailing pulse, and the pulse spacing of $2.6 \mu$ s corresponds well with the prediction obtained from Eq. 2. Figure $11 \mathrm{~b}$ shows the second echo, scattered twice by the crack and once by the plate edge, arriving near $206 \mu \mathrm{s}$. The leading pulse is scarcely detectable, and the third pulse (the second trailing pulse) is largest in magnitude, slightly greater than the fourth pulse. Figure 11c shows the third echo, and with the sensitivity setting used in recording that plot the first arrival occurs near $315 \mu$ s and the strongest pulses in this train are respectively the third and the fourth in the train. This suggests that the original leading pulse and first trailing pulse were below the threshold of observation, that the first arrival was originally the second trailing (third overall) pulse, and that the strongest pulses send in this record were originally the fifth and sixth overall. (Figure $11 \mathrm{c}$ is complicated somewhat by the fact that an echo from the far edge would arrive near this same time, diffracted on its return path by the crack.) Figure $11 \mathrm{~d}$ shows the fourth and fifth echoes, arriving roughly near 420 and $525 \mu \mathrm{s}$, but we do not attempt to trace the identity of the individual pulses in those trains.

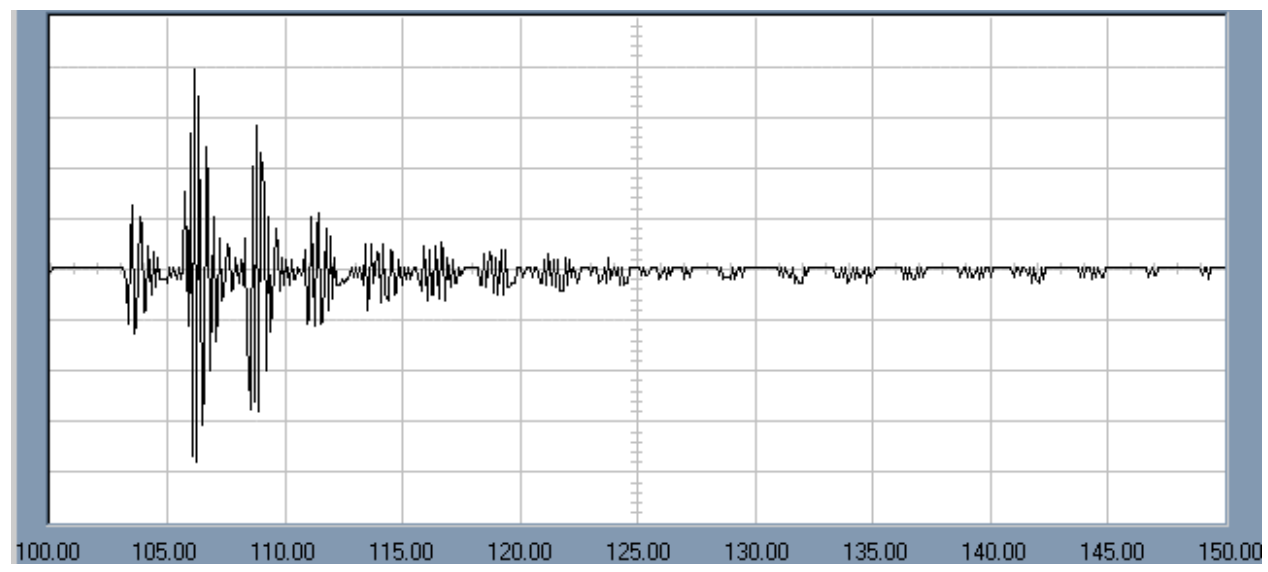

Figure 11a. First echo from crack arrives near $103 \mu$ s, trailing pulses observed at approximately $2.6 \mu \mathrm{s}$ 


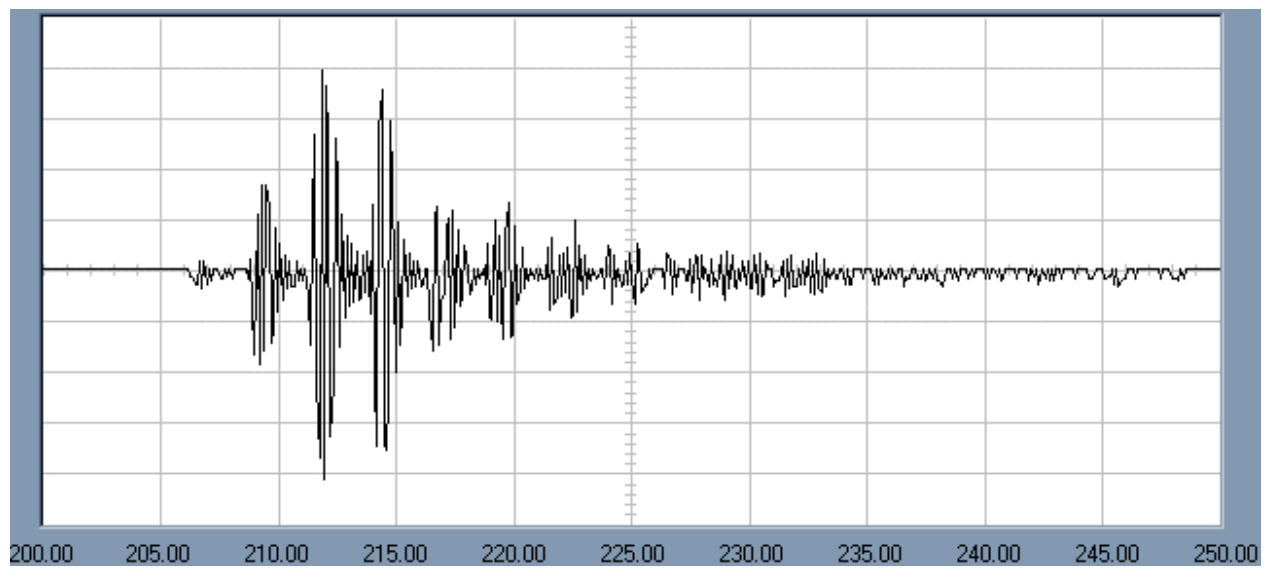

Figure 11b. Second echo from crack arrives near $206 \mu$ s

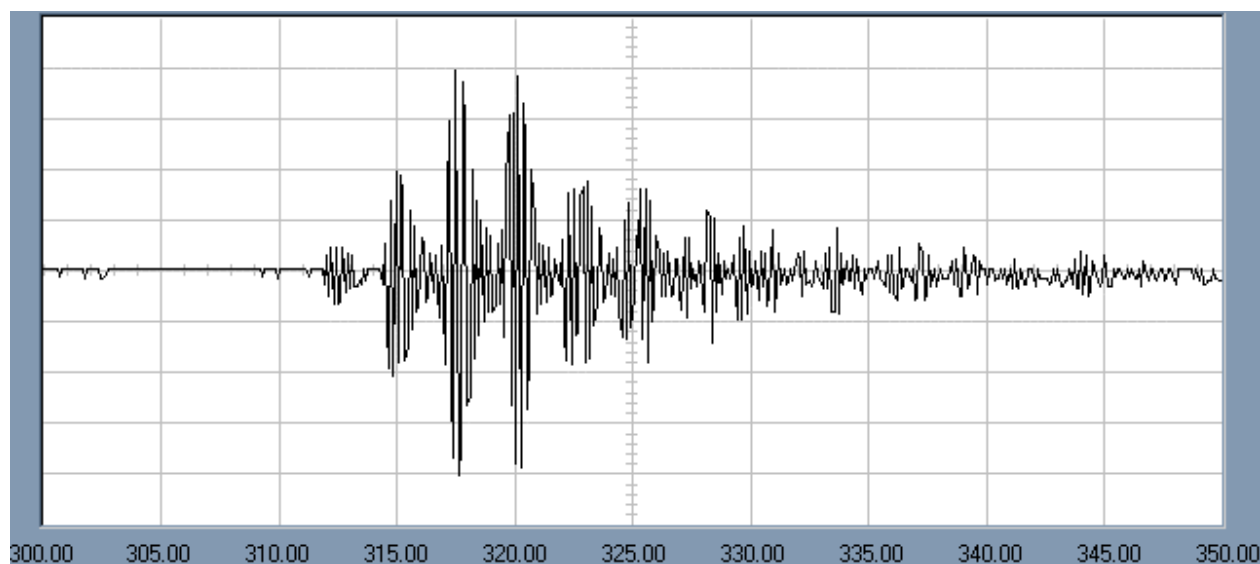

Figure 11c. Third echo from crack arrives near $315 \mu$ s (roughly coincident with echo from far edge of plate)

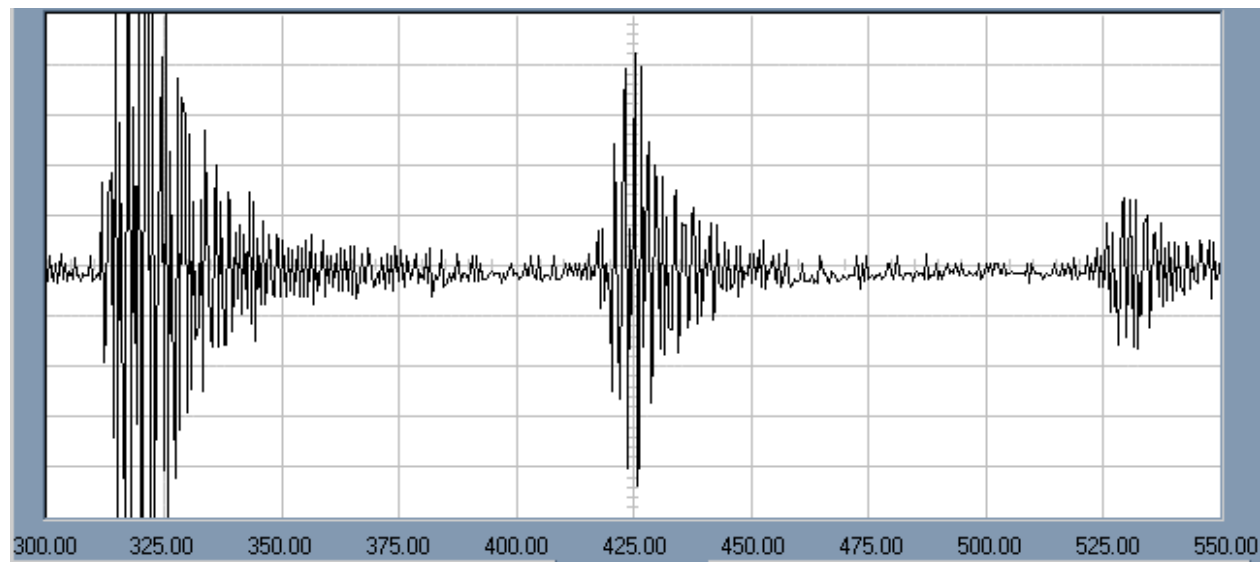

Figure 11d. Fourth and fifth echoes from crack arrive near 420 and $525 \mu \mathrm{s}$, respectively (note change in time scale) 
The experiment depicted in Figure 10 was repeated with the probe placed at the left edge of the plate, $60.5 \mathrm{~cm}$ from the crack location. Figure 12a shows the first echo arriving near $206 \mu \mathrm{s}$, with the leading pulse scarcely detectable and the third pulse (the second trailing pulse) largest in magnitude, slightly greater than the fourth pulse; this essentially reproduces the record shown in Figure 11b, which was a second echo but with the same total path length. Figure $12 \mathrm{~b}$ shows an echo arriving near $315 \mu \mathrm{s}$, representing a reflection from the far edge, which would have been diffracted when passing the crack location, and an echo arriving $415 \mu \mathrm{s}$,, representing the second echo from the crack. We note that the echo from the relatively small crack is easily detected even after multiple echoes and even after the wave has traveled a path length of almost $3 \mathrm{~m}$. We observe that the crack could have been detected at even greater distances; our experiment was limited not by amplification or sensitivity, but by the time limit of signal acquisition, slightly more than $600 \mu$ s.

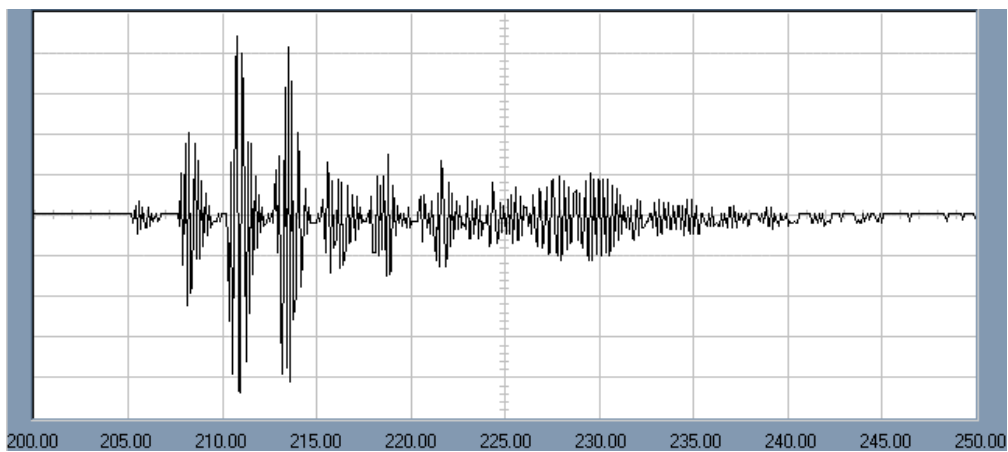

Figure 12a. First echo from crack arrives near $206 \mu$ s (transducer has been moved to left edge of plate in Figure 10)

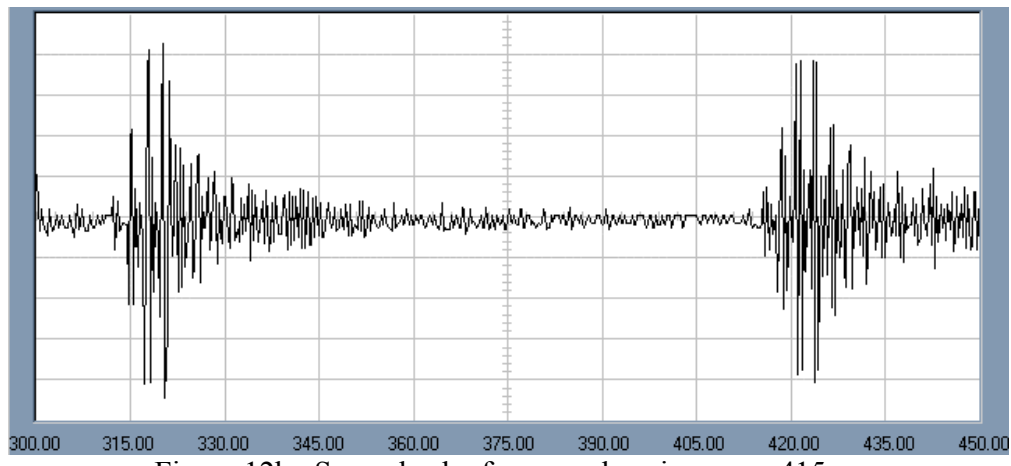

Figure 12b. Second echo from crack arrives near $415 \mu \mathrm{s}$

Finally, crack detection was tested on a large plate $19 \mathrm{~mm}$ thick with a through-thickness crack, $6 \mathrm{~cm}$ long, $96.4 \mathrm{~cm}$ from the plate edge. Figure 13 shows the first echo arriving near $327 \mu$ s, which corresponds precisely with the travel time for a longitudinal pulse and therefore is evidence that the pulse is the original leading pulse. The spacing between trailing pulses is approximately $5 \mu \mathrm{s}$, as observed earlier in Figure 2 with a plate of the same 19-mm thickness.

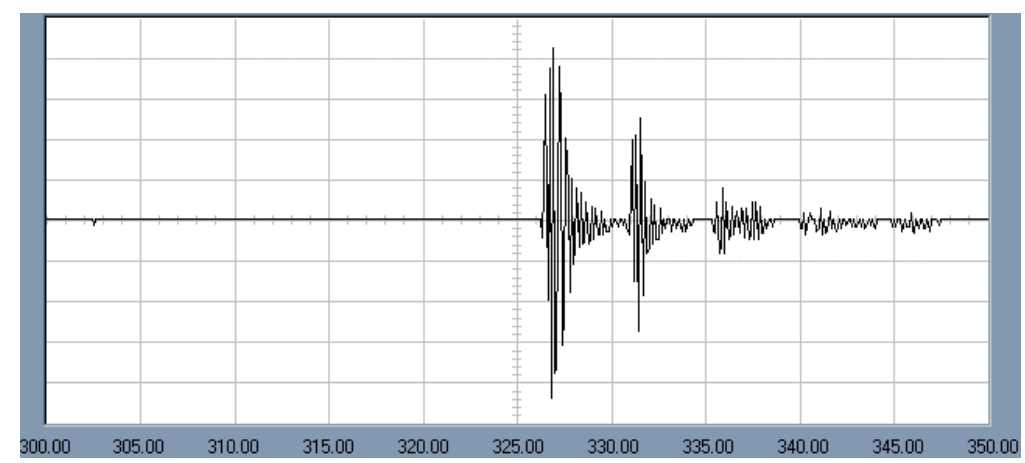

Figure 13. First echo from crack arrives near $327 \mu$ s (crack $96.4 \mathrm{~cm}$ from plate edge, $19 \mathrm{~mm}$ plate thickness) 


\section{SUMMARY AND CONCLUSIONS}

We have reviewed the generation of waves in plates by force excitation normal to a plate edge. Simulations and experiments both show that multiple Lamb modes are created at low fd products, while for fd greater than $15 \mathrm{MHz}-\mathrm{mm}$ a nearly longitudinal pulse is generated which is followed by several trailing pulses. During propagation, energy is transferred from the leading pulse to trailing pulses by boundary reflections from the surfaces of the plate. Simulations and experiments both show that the pulse train retains its integrity over distances of at least $1 \mathrm{~m}$, and subsequent experiments show the pulse train to retain its integrity over distances greater than $3 \mathrm{~m}$. We suggest that these nearlylongitudinal waves are attractive as an alternative to Lamb waves for the detection of defects in plate-like structures. An important advantage of the nearly-longitudinal modes is the shorter wavelength which will result in enhanced scattering from small defects.

We simulated the scattering of a pulse train by a part-thickness slot in a plate $19 \mathrm{~mm}$ thick, in which both the transmitted and reflected pulse trains show no sign of mode conversion to Lamb waves, and then obtained experimental confirmation of that observation. Finally, we studied pulse-echo signals reflected from a small through-thickness flaw in plates 9.5 and $19 \mathrm{~mm}$ thick, tested with a commercial ultrasonic flaw detection system using a $5.0 \mathrm{MHz}$ probe. Echoes were unambiguous even after multiple reflections and even with a total path length approaching $3 \mathrm{~m}$.

It is instructive to compare the nearly-longitudinal transients as they are described here to Lamb waves (harmonic waves) as they are most commonly described mathematically, with particular attention to practical implications for flaw detection. Setting aside the influence of material attenuation, which is relatively modest in materials such as steel or aluminum, and temporarily setting aside the geometric spreading of the wavefront, a Lamb wave can be viewed as a harmonic wave, extending indefinitely in time and space. The nearly-longitudinal wave described here is a transient disturbance that sheds its energy into an ever-changing train of trailing pulses. However, the practical question is not whether it will propagate to infinity, but whether it will propagate far enough to permit useful flaw detection. The measurements summarized in Figures 12 and 13 show that an ultrasonic pulse can propagate considerable distances, approaching $3 \mathrm{~m}$, with sufficient energy and preservation of pulse shape to provide unambiguous pulse-echo flaw detection. The short wavelength of the relatively high-frequency pulse forms an unambiguous echo from flaw sizes of practical interest, and the absence of multiple modes makes it easy to interpret the echoes, in contrast to the challenge when multiple Lamb wave modes are present. Whereas Lamb waves in plates are correctly termed guided waves, the nearly-longitudinal wave, excited at relatively high frequency by an edge-mounted transducer, is also a guided transient disturbance with great practical utility.

\section{ACKNOWLEDGEMENTS}

The authors gratefully acknowledge support from Bombardier Total Transit Systems and the Pennsylvania Infrastructure Technology Alliance and from the National Science Foundation under grant CMS-0329880. Any opinions, findings, and conclusions or recommendations expressed in this material are those of the authors and do not necessarily reflect the views of the National Science Foundation.

\section{REFERENCES}

1. J.-B. Ihn and F.-K. Chang,"Detection and monitoring of hidden fatigue crack growth using a built-in piezoelectric sensor/actuator network: I. Diagnostics," Smart Mater. Struct. 13, 609-620, 2004.

2. Zhu, W., and Rose, J. L., "Lamb wave generation and reception with time-delay periodic linear arrays: A BEM simulation and experimental study," IEEE Trans. Ultrasonics, Ferroelectrics and Freq. Ctt., 46, 654-664, 1999.

3. Giurgiutiu, G., "Lamb Wave Generation with Piezoelectric Wafer Active Sensors for Structural Health Monitoring," Proc. SPIE-Smart Structures and Materials 2003: Smart Structures and Integrated Systems, 5056, 111-122, 2003.

4. Greve, D. W., Zheng, P., and Oppenheim, I. J., "Excitation of longitudinal and Lamb waves in plates by edgemounted transducers," Proc. IEEE Ultrasonics Conference, New York, October 2007.

5. Krautkramer, J., and Krautkramer, H., Ultrasonic Testing of Materials, 4th fully rev. ed., Springer-Verlag, 1990. 